\title{
Commutation Relations in Mesoscopic Electric Circuits
}

\author{
You-Quan Li \\ Institut für Physik, Universität Augsburg, D-86135 Augsburg, Germany \\ and Department of Physics, Zhejiang University, Hangzhou 310027, China \\ email:yqli@physik.uni-augsburg.de
}

\begin{abstract}
In the talk, I briefly demonstrate the quantum theory for mesoscopic electric circuits and its applications. In the theory, the importance of the charge discreteness in a mesoscopic electric circuit is addressed. As a result, a new kind of commutation relation for electric charge and current occurred inevitably. The charge representation, canonical current representation and pseudo-current representation are discussed extensively. It not only provides a concrete realization of mathematical models which discuss the space quantization in high energy physics and quantum gravity but also presents a sequence of applications in condensed matter physics from a different point of view. A possible generalization to coupled circuits is also proposed.
\end{abstract}

\section{INTRODUCTION}

The dramatic achievement in nanotechnology has aroused tremendous developments in experimental physics in mesoscopic scale. Miniaturization of integrated circuits is undoubtedly a persistent trend for electronic device community. A theory for mesoscopic circuits was proposed by $\mathrm{Li}$ and Chen, in which the charge discreteness is first introduced in the quantization of electric circuits [1]. The possibility of space-time discreteness was early considered by Snyder [2] who indicated that the Lorentz invariance do not exclude quantized (discrete) space-time, it was also argued by Li [3] from the finiteness of the observed universe. S. Mantecinos, I. Saavedra, and O. Kunstmann [4] discussed the commutation relations of [2] arguing that it may be related to physics in high energy scale $\left(10^{9}-10^{12} \mathrm{eV}\right)$. Numerous attempts to the argument of exiting minimal position uncertainty were made [5] on the basis of various considerations in string theory as well as in quantum gravity. Actually, the approach of [1] not only provides a concrete realization of mathematical models for exploring the space quantization in high energy physics and quantum gravity but also presents a sequence of applications in condensed matter physics from a different point of view. For example, the persistent current is solved by regarding the mesoscopic metal ring as the circuit of a pure L-design. Application of the theory to a pure C-design gives rise to the Coulomb blockade solution [6]. 


\section{BASIC DEFINITIONS AND COMMUTATION RELATIONS}

Let $\hat{q}$ denote for the charge operator, and $\hat{p}$ for the canonical conjugation of the charge satisfying $[\hat{q}, \hat{p}]=i \hbar$. We call $\hat{p}$ the canonical current operator since it is not only the canonical conjugation of charge but also the current operator in the quantization approach [7] where the charge was considered as a continuous variable. Taking into account of the discreteness of electronic charge in quantization procedure, we must impose that the eigenvalues of the self-adjoint operator $\hat{q}$ take discrete values [1], i.e. $\hat{q}|n\rangle=n q_{e}|n\rangle$ where $n \in \mathbb{Z}$ (set of integers) and $q_{e}=$ $1.602 \times 10^{-19}$ coulomb, the elementary electric charge. We therefore introduce a minimum 'shift operator' $\hat{Q}:=e^{i q_{e} \hat{p} / \hbar}$ in charge space, which satisfies [1]

$$
[\hat{q}, \hat{Q}]=-q_{e} \hat{Q}, \quad \hat{Q}^{-1}=\hat{Q}^{\dagger} .
$$

These relations determine the structure of the whole Fock space, accordingly, $\hat{Q}^{+}|n\rangle=e^{i \alpha_{n+1}}|n+1\rangle, \hat{Q}|n\rangle=e^{-i \alpha_{n}}|n-1\rangle$ where $\alpha_{n}$ 's being undetermined phases. The Fock space for the algebra (1) differs from the well known Fock space for the Heisenberg-Weyl algebra because the spectrum of the former is isomorphic to the set of integers $\mathbb{Z}$ but that of the later is isomorphic to the set of non-negative integers $\mathbb{Z}^{+}+\{0\}$. Since $\{|n\rangle \mid n \in \mathbb{Z}\}$ spans a Hilbert space and $\hat{q}$ is self-adjoint, both the completeness $\sum_{n \in \mathbf{Z}}|n\rangle\langle n|=1$ and the orthogonality $\langle n \mid m\rangle=\delta_{n m}$ faithful.

The quasi-current $\hat{J}$ for a mesoscopic circuit is defined by $\hat{J}=-i \hbar\left(\hat{Q}^{1 / 2}-\right.$ $\left.\hat{Q}^{-1 / 2}\right) / q_{e}$ which reduces to the canonical current in the limit $q_{e} \rightarrow 0$. The Hamiltonian of a mesoscopic LC-circuit is given by [1]

$$
\hat{H}=-\frac{\hbar^{2}}{2 L} \hat{J}^{2}+\frac{1}{2 C} \hat{q}^{2}+\varepsilon \hat{q},
$$

where $\varepsilon$ stands for the voltage source, $L$ for inductance, and $C$ for capacity of the circuit. Using eq.(1) we easily obtain the new commutation relations for the quasi-current operator,

$$
[\hat{q}, \hat{J}]=i \frac{\hbar}{2} \hat{K}, \quad[\hat{q}, \hat{K}]=-i \frac{q_{e}^{2}}{2 \hbar} \hat{J}
$$

where an auxiliary operator $\hat{K}=\hat{Q}^{1 / 2}+\hat{Q}^{-1 / 2}$ is introduced. Obviously eq.(3) obeys the $\mathrm{SU}(2)$ algebra after rescaling the operators. In terms of $\hat{K}$ and $\hat{J}$ we can define a useful operator $\hat{P}=\hat{J} \hat{K}^{-1}$ which we call the pseudo-current operator. Obviously the pseudo-current also reduces to canonical current in the limit $q_{e} \rightarrow 0$. With the help of (3), we obtain the following commutation relations,

$$
[\hat{q}, \hat{P}]=i \hbar\left(1+\left(\frac{q_{e}}{2 \hbar}\right)^{2} \hat{P}^{2}\right) .
$$

Similar kind of commutation relation was considered earlier in [2] in searching the possibility of space-time discreteness. From the commutation relation (4) one will 
have a uncertainty relation [4,8] for the charge and pseudo-current [1], which is different from the conventional Heisenberg uncertainty relation.

The definition of physical current $\hat{I}$ arises from the Heisenberg equation $\hat{I}=$ $d \hat{q} / d t=(1 / i \hbar)[\hat{q}, \hat{H}]$. For the $L C$-design circuit, one can immediately obtain [9],

$$
\hat{I}=-i \frac{\hbar}{2 q_{e} L}\left(\hat{Q}-\hat{Q}^{\dagger}\right)
$$

\section{PSEUDO-CURRENT REPRESENTATION}

We consider the pseudo-current representation $\hat{P}|\eta\rangle=\eta|\eta\rangle$. The differential realization of commutation relation (4) is given by [2]

$$
\hat{P}=\eta, \quad \hat{q}=i \hbar\left(1+\left(\frac{q_{e}}{2 \hbar} \eta\right)^{2}\right) \frac{\partial}{\partial \eta}
$$

Obviously, $\int d \eta \psi^{*}(\eta) \hat{q} \phi(\eta)$ fails in guaranteeing the charge operator being selfadjoint. The factor $\left(1+\left(\eta q_{e} / 2 \hbar\right)^{2}\right)^{-1}$ in the measure on the pseudo-current space is therefore required [8] to cancel the corresponding factor of $\hat{q}$ in this representation. The inner product must be so defined,

$$
\langle\psi \mid \phi\rangle=\int_{-\infty}^{\infty} \frac{d \eta}{1+\left(\frac{q_{e}}{2 \hbar} \eta\right)^{2}} \psi^{*}(\eta) \phi(\eta)
$$

that both $\hat{q}$ and $\hat{P}$ could be self-adjoint.

The completeness is given by

$$
\int_{-\infty}^{\infty} \frac{d \eta}{1+\left(\frac{q_{e}}{2 \hbar} \eta\right)^{2}}|\eta\rangle\langle\eta|=1 .
$$

Consequently, the inner product of two eigenstates of the pseudo-current operator yields

$$
\left\langle\eta^{\prime} \mid \eta\right\rangle=\left(1+\left(\frac{q_{e}}{2 \hbar} \eta\right)^{2}\right) \delta\left(\eta-\eta^{\prime}\right)
$$

The eigen-equation of charge operator in this representation reads

$$
i \hbar\left(1+\left(\frac{q_{e}}{2 \hbar} \eta\right)^{2}\right) \frac{\partial}{\partial \eta} \psi_{q}(\eta)=q \psi_{q}(\eta)
$$

where $\psi_{q}(\eta):=\left\langle\eta \mid \psi_{q}\right\rangle$. The deferential equation (10) is solved by

$$
\psi_{q}(\eta)=\left(\frac{q_{e}}{2 \pi \hbar}\right)^{1 / 2} \exp \left(-i q \frac{2}{q_{e}} \tan ^{-1}\left(\frac{q_{e}}{2 \hbar} \eta\right)\right)
$$


which has been normalized. It is interesting to evaluate their inner product

$$
\left\langle\psi_{q} \mid \psi_{q^{\prime}}\right\rangle=\frac{q_{e}}{\left(q^{\prime}-q\right) \pi} \sin \left(\frac{q^{\prime}-q}{q_{e}} \pi\right) .
$$

This clearly brings about a orthogonal catastrophe because the eigenstate of a selfadjoint operator with different eigenvalues must be mutually orthogonal. Actually, it can be avoided provided that $q^{\prime}-q=n q_{e}$. We conclude that the electric charge must be quantized (other eigenvalues are not physically permitted).

A natural choice is $q=n q_{e}$, then the transformation from charge representation to pseudo-current representation is easily derived,

$$
\begin{aligned}
\langle\eta \mid \psi\rangle & =\sum_{n=-\infty}^{\infty}\langle\eta \mid n\rangle\langle n \mid \psi\rangle \\
& =\left(\frac{q_{e}}{2 \pi \hbar}\right)^{1 / 2} \sum_{n=-\infty}^{\infty}\langle n \mid \psi\rangle e^{-i n \Theta(\eta)},
\end{aligned}
$$

where $\Theta(x)=2 \tan ^{-1}\left(x q_{e} / 2 \hbar\right)$. Multiplying (13) by $e^{i n^{\prime} \Theta(\eta)} /\left[1+\left(\eta q_{e} / 2 \hbar\right)^{2}\right]$ and integrating with respect to $\eta$ give rise to the inverse transformation:

$$
\langle n \mid \psi\rangle=\left(\frac{q_{e}}{2 \pi \hbar}\right)^{1 / 2} \int_{-\infty}^{\infty} \frac{d \eta}{1+\left(\frac{q_{e}}{2 \hbar} \eta\right)^{2}} e^{i n \Theta(\eta)}\langle\eta \mid \psi\rangle .
$$

\section{CANONICAL CURRENT REPRESENTATION}

In the canonical current space $\hat{p}|p\rangle=p|p\rangle$, the $\hat{q}$ and $\hat{p}$ are realized by $\hat{p}=p$, $\hat{q}=i \hbar \partial / \partial p$. The eigen-equation of charge operator is,

$$
i \hbar \frac{\partial}{\partial p} \psi_{q}(p)=q \psi_{q}(p)
$$

which is solved by plane waves $\psi_{q}(p)=e^{-i q p / \hbar}$. Obviously, the periodic condition in p-space, $\psi_{q}\left(p+2 \pi \hbar / q_{e}\right)=\psi_{q}(p)$ should be imposed so that the charge is quantized (discrete) $q / q_{e}=n$, consequently,

$$
\psi_{n}(p)=\langle p \mid n\rangle=e^{-i n p q_{e} / \hbar}
$$

The transformation from charge representation to canonical current representation is easily obtained,

$$
\begin{aligned}
\langle p \mid \psi\rangle & =\sum_{n=-\infty}^{\infty}\langle p \mid n\rangle\langle n \mid \psi\rangle \\
& =\sum_{n=-\infty}^{\infty} e^{-i n p q_{e} / \hbar}\langle n \mid \psi\rangle .
\end{aligned}
$$


Multiplying eq.(16) with $e^{i n^{\prime} p q_{e} / \hbar}$ and integrating with respect to $p$, we get the inverse transformation, canonical current representation to charge representation:

$$
\langle n \mid \psi\rangle=\frac{q_{e}}{2 \pi \hbar} \int_{-\pi \hbar / q_{e}}^{\pi \hbar / q_{e}} d p\langle p \mid \psi\rangle e^{i n p q_{e} / \hbar} .
$$

In the p-space, the Hamiltonian for a mesoscopic LC-design circuit becomes

$$
\hat{H}=-\frac{\hbar^{2}}{q_{e}^{2} L}\left[\cos \left(\frac{q_{e}}{\hbar} p\right)-1\right]-\frac{\hbar^{2}}{2 C}\left(\frac{\partial}{\partial p}+i \frac{C}{\hbar} \varepsilon\right)^{2}-\frac{C}{2} \varepsilon^{2} .
$$

The advantage of the canonical current representation is that the Schrödinger equation for the Hamiltonian (18) becomes the standard Mathieu equation after a unitary transformation. The wave function was solved in terms of periodic Mathieu functions, and the energy spectrum was expressed by the eigenvalues of Mathieu equation. The details can be find in [1].

\section{APPLICATIONS}

In Coulomb blockade experiments, the mesoscopic capacity may be relatively very small (about $10^{-8} \mathrm{~F}$ ) but the inductance of a macroscopic circuit connecting to a source is relatively large because it is proportional to the area which the circuit spans. We can neglect the term reversely proportional to $L$ in (2), and study the equation for a pure C-design. Because a mesoscopic metal ring can be regarded as a pure L-design, we can also study the persistent current on a mesoscopic ring.

\section{Coulomb blockade}

The Schrödinger equation for a pure C-design reads

$$
\left(\frac{1}{2 C} \hat{q}^{2}-\varepsilon \hat{q}\right)|\psi\rangle=E|\psi\rangle,
$$

where $\varepsilon$ is an adiabatic voltage source. The Hamiltonian and charge operator commute each other, so $|n\rangle$ is the eigenstate with energy $E=\left(n q_{e}-C \varepsilon\right)^{2} / 2 C-$ $C \varepsilon^{2} / 2$, where both the charge quantum number and the voltage source are involved. The relation between charge $q$ and the voltage $\varepsilon$ for the ground state is given by

$$
q=\sum_{m=0}^{\infty}\left\{\theta\left[\varepsilon-\left(m+\frac{1}{2}\right) \frac{q_{e}}{C}\right]-\theta\left[-\varepsilon-\left(m+\frac{1}{2}\right) \frac{q_{e}}{C}\right]\right\} q_{e}
$$

where $\theta(x)$ is the step function. The corresponding eigenstate is

$$
|\psi(\varepsilon)\rangle_{\text {ground }}=\sum_{m=-\infty}^{\infty}\left\{\theta\left[\varepsilon-\left(m-\frac{1}{2}\right) \frac{q_{e}}{C}\right]-\theta\left[\varepsilon-\left(m+\frac{1}{2}\right) \frac{q_{e}}{C}\right]\right\}|m\rangle .
$$


The dependence of the current on time is obtained by taking derivative

$$
\frac{d q}{d t}=\sum_{m=0}^{\infty} q_{e}\left\{\delta\left[\varepsilon-\left(m+\frac{1}{2}\right) \frac{q_{e}}{C}\right]+\delta\left[\varepsilon+\left(m+\frac{1}{2}\right) \frac{q_{e}}{C}\right]\right\} \frac{d \varepsilon}{d t} .
$$

Clearly, the current is of a form of sharp pulses which occurs periodically (with periodicity $\left.q_{e} / C\right)$ according to the changes of voltage.

\section{Persistent current}

The Schrödinger equation for a pure L-design in the presence of magnetic flux is given by,

$$
-\frac{\hbar^{2}}{2 q_{e}^{2} L}\left(e^{-i \frac{q_{e}}{\hbar} \phi} \hat{Q}+e^{i \frac{q_{e}}{\hbar} \phi} \hat{Q}^{+}-2\right)|\psi\rangle=E|\psi\rangle .
$$

It is obtained on the basis of gauge covariance [1]. The eigenstates can be simultaneous eigenstates of $\hat{p}$, eq. (23) is solved by the eigenstate $|p\rangle=\sum_{n \in \mathbf{Z}} \kappa_{n} e^{i n q_{e} p / \hbar}|n\rangle$ $\left(\kappa_{n}:=\exp \left(i \sum_{j=1}^{n} \alpha_{j}\right)\right)$ with the energy spectrum:

$$
E(p, \phi)=\frac{2 \hbar^{2}}{q_{e}^{2} L} \sin ^{2}\left(\frac{q_{e}}{2 \hbar}(p-\phi)\right) .
$$

It oscillates with respect to $\phi$ or $p$. Differing from the usual classical pure Ldesign, the energy of a mesoscopic quantum pure L-design can not be large than $2 \hbar^{2} / q_{e}^{2} L$. Clearly, the lowest energy states are those states with $p=\phi+n h / q_{e}$, the eigenvalues of the electric current $\hat{I}$ of ground state can be obtained [1]. The electric current on a mesoscopic circuit of pure L-design is not null in the presence of a magnetic flux (except $\phi=n h / q_{e}$ ). This is a quantum characteristic property. The persistent current in a mesoscopic L-design is an observable quantity periodically depending on the flux $\phi$. In terms of the inductance of mesoscopic metal ring, $L=8 \pi r\left(\frac{1}{2} \ln \frac{8 r}{a}-1\right)$ where $r$ is the radius of the ring and $a$ is the radius of the metal wire, the formula for persistent current on a mesoscopic ring is obtained

$$
I(\phi)=\frac{\hbar}{8 \pi r\left(\frac{1}{2} \ln \frac{8 r}{a}-1\right) q_{e}} \sin \left(\frac{q_{e}}{\hbar} \phi\right) .
$$

Differing from the conventional formulation of the persistent current on the basis of quantum dynamics for electrons, This formulation presented a method from a new point of view. Formally, the $I(\phi)$ is a sine function with periodicity of $\phi_{0}=h / q_{e}$. But either the model that the electrons move freely in an ideal ring [10], or the model that the electrons have hard-core interactions between them [11] can only give the sawtooth-type periodicity. Obviously, the sawtooth-type function is only the limit case for $q_{e} / \hbar \rightarrow 0$. 


\section{COUPLED CIRCUITS}

The above discussions are based on a single mesoscopic circuit. Let us consider the case that several circuits coupled to each other by mutual inductances, associated capacities or any other kind of coupling. Because of quantum tunneling and quantum fluctuations, the charges in individual circuit is no longer precisely measurable. The charge on each circuit are not good quantum numbers, and therefore the charge operators $\hat{q}_{j}$ are expected to be noncommutative. A natural generalization of the formulation for single circuit is easily carried out in the pseudo-current representation, namely,

$$
\hat{P}_{j}=\eta_{j}, \quad \hat{q}_{j}=i \hbar\left(1+\left(\frac{q_{e}}{2 \hbar}\right)^{2} \vec{\eta}^{2}\right) \frac{\partial}{\partial \eta_{j}} .
$$

It is easily to obtain the following commutation relation,

$$
\left[\hat{q}_{i}, \hat{q}_{j}\right]=i \frac{q_{e}^{2}}{2 \hbar} M_{i j}
$$

where $M_{i j}:=\hat{P}_{i} \hat{q}_{j}-\hat{P}_{j} \hat{q}_{i}$. The charge operators for individual circuit are generally noncommutative for the non-vanishing $\left\langle M_{i j}\right\rangle$. This provides a concrete physical example of noncommutative geometry. Further studies are in progress.

\section{ACKNOWLEDGMENT}

The work is supported by AvH Stiftung, NSFC-19975040 and EYFC98. I would like to thank J.C. Flores for communication and reference. Interesting discussions with O.W. Greenburg, M.S. Plyushchay, A. Solomon, E.C.G. Sudarshan, A. Zee et.al. are also acknowledged.

\section{REFERENCES}

1. Li Y.Q. and Chen B., Phys. Rev. B 53, 4027-4032 (1996).

2. Snyder H.S., Phys. Rev. 71, 38-41 (1947).

3. Li Y.Q., Commun. Theor. Phys. 24, 255-256 (1995).

4. Mantecinos S., Saavedra I., and Kunstmann O., Phys. Lett. A 109, 139-142 (1985).

5. Garay L.J., Int. J. Mod. Phys. A 10, 145-166 (1995) and references therein.

6. Li Y.Q., "Mesoscopic quantum circuit theory to persistent current and Coulomb blockade" in Proceedings of the 5th Wigner Symposium, edited by P. Kasperkovitz \& D. Grau, World. Sci., Singpore 1998 pp.307-310.

7. Louisell W.H., Quantum Statistical Properties of Radiation, John Wiley, New York 1973.

8. Kempf A., Mangano G., and Mann K.B., Phys. Rev. D 52, 1108-1118 (1995).

9. Flores J.C., e-print cond-mat/9908012.

10. Cheung H.F., Gefen Y., Riedel E.K., et. al., Phys. Rev. B, 37, 6050-6062 (1988).

11. Li Y.Q. and Ma Z.S., J. Phys. Soc. Jpn., 65, 1519-1522 (1996). 